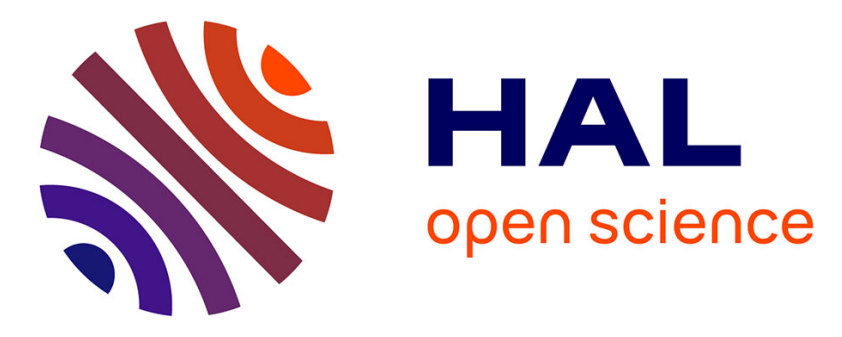

\title{
Virtual Reality as a Tool to Learn Interpersonal Coordination: Example of Team Rowing
}

\author{
Manuel Varlet, Alessandro Filippeschi, Grégory Ben-Sadoun, Mickael Ratto, \\ Ludovic Marin, Emanuele Ruffaldi, Benoît Bardy
}

\section{- To cite this version:}

Manuel Varlet, Alessandro Filippeschi, Grégory Ben-Sadoun, Mickael Ratto, Ludovic Marin, et al.. Virtual Reality as a Tool to Learn Interpersonal Coordination: Example of Team Rowing. Presence: Teleoperators and Virtual Environments, 2013, 22 (3), pp.202-215. 10.1162/PRES_a_00151. hal02432957

\section{HAL Id: hal-02432957 \\ https://hal.umontpellier.fr/hal-02432957}

Submitted on 8 Jan 2020

HAL is a multi-disciplinary open access archive for the deposit and dissemination of scientific research documents, whether they are published or not. The documents may come from teaching and research institutions in France or abroad, or from public or private research centers.
L'archive ouverte pluridisciplinaire HAL, est destinée au dépôt et à la diffusion de documents scientifiques de niveau recherche, publiés ou non, émanant des établissements d'enseignement et de recherche français ou étrangers, des laboratoires publics ou privés. 


\section{Manuel Varlet*}

Movement to Health Laboratory

EuroMov

Montpellier-I University

Montpellier, France

\section{Alessandro Filippeschi}

PERCRO Lab

Scuola Superiore S. Anna

Pisa, Italy

\section{Grégory Ben-sadoun \\ Mickael Ratto \\ Ludovic Marin}

Movement to Health Laboratory

EuroMov

Montpellier-I University

Montpellier, France

\section{Emanuele Ruffaldi}

PERCRO Lab

Scuola Superiore S. Anna

Pisa, Italy

\section{Benoît G. Bardy}

PERCRO Lab

Scuola Superiore S. Anna

Pisa, Italy, and

Institut Universitaire de France

Presence, Vol. 22, No. 3, Summer 2013, 202-215 doi: 10.1 162/PRES_a_00 I 51

( 2014 by the Massachusetts Institute of Technology

\section{Virtual Reality as a Tool to Learn \\ Interpersonal Coordination: \\ Example of Team Rowing}

\begin{abstract}
The success of interpersonal activities strongly depends on the coordination between our movements and those of others. Learning to coordinate with other people requires a long training time and is often limited by the difficulty of having people available at the same time and of giving them accurate and real-time feedback about their coordination. The goal of the present study was to determine in an indoor team rowing situation whether virtual-reality and motion-capture technologies can help the acquisition of interpersonal coordination. More specifically, we investigated the possibility for participants to ( 1 ) learn the skill of interpersonal coordination when training with a virtual teammate, (2) accelerate learning with real-time visual feedback, and (3) transfer this skill to synchronizing situations with a real teammate. Our results show that participants improved their coordination with both virtual and real teammates, and that this improvement was better for participants who received the feedback. Generally, our results demonstrate the interest of virtual reality for learning the coordination with other people; further, our results open promising training perspectives for team rowing but also for several other interpersonal activities.
\end{abstract}

\section{Introduction}

The coordination of our movements with other people plays a crucial role in the success of interpersonal activities. This the case in sports such as dancing or rowing but also in everyday life situations, for example, when coordinating with someone else to move a heavy object (e.g., Baudouin \& Hawkins, 2002; Hill, 2002; Marsh, Richardson, Baron, \& Schmidt, 2006; Marsh, Richardson, \& Schmidt, 2009; Richardson, Marsh, \& Baron, 2007). Interpersonal coordination also influences the success of everyday social interactions by enhancing cognitive activities of the actors such as their feeling of cohesion, connectedness, social rapport, and the efficiency of their communication (Bernieri, 1988; Bernieri, Davis, Rosenthal, \& Knee, 1994; Chartrand \& Bargh, 1999; Hove \& Risen, 2009; Lakin \& Chartrand, 2003; Richardson, Dale, \& Kirkham, 2007; Tickle-Degnen \& Rosenthal, 1990). Accordingly, improving such coordination is not only of interest in sports activities but also for people suffering from social interaction disorders such as schizophrenic patients (Brune et al., 2008; Kupper, Ramseyer, Hoffmann, Kalbermatten, \& Tschacher, 2010; Varlet, Marin, Raffard, et al., 2012). Although the visuomotor processes underlying

\footnotetext{
*Correspondence to manuel.varlet@gmail.com.
} 
such coordination are well understood (see Schmidt \& Richardson, 2008, for a review), no learning protocols have yet been developed. Following researchers' growing interest in studying interpersonal activities, developing learning or relearning protocols represents an exciting challenge for researchers, coaches, and clinicians. In the current study, we investigated whether virtual reality can be used as a tool to facilitate the learning of interpersonal coordination, an investigation illustrated throughout this paper more specifically with the example of learning team rowing coordination.

The interest of virtual reality technologies to better understand and improve perceptuo-motor skills and sports performance has been investigated in several studies (c.g., Bideau ct al., 2003, 2010; Lammfromm \& Gopher, 2011; Ruffaldi et al., 2011; Todorov, Shadmehr, \& Bizzi, 1997; Vignais et al., 2009; Vignais, Bidean, ct al., 2010; Vignais, Kulpa, et al., 2010; Zaal \& Bootsma, 2011). However, mostly due to the difficulty of transferring the skills acquired in the virtual world to the real world, successful learning has rarely been reported (e.g., Lammfromm \& Gopher, 2011; Todorov et al., 1997). The principal difficulty in such sports simulations is probably to render reality/crucial interactions in a way that enables transfer. A potential limitation of transferring interpersonal coordination from virtual to real situations relates to the differences between virtual humans and real humans with whom we usually interact. In fact, it has been shown that visuomotor processes underlying the coordination with human and nonhuman stimuli are different, and that the coordination might be facilitated when movements are performed by a human, an effect that tends to vanish when appearance and movements of the artificial agent become closer to those of a human (Blakemore \& Frith, 2005; Kilner, Paulignan, \& Blakemore, 2003; Oztop, Franklin, Chaminade, \& Gordon, 2005; Press, Bird, Flach, \& Heyes, 2005; Stanley, Gowen, \& Miall, 2007). However, it remains largely unknown whether learning an interpersonal coordination pattern with a virtual human is of any help for coordination with a real human.

Overcoming such potential limitations motivated the current study. Moreover, we expected that virtual reality would offer conditions to the trainees that are not avail- able with real practice and would therefore facilitate learning. Previous research using real-time motion cap ture technology has shown the possibility of giving feedback in the virtual world to the trainee which can accelerate learning and would be very hard to render in the real world (Chan, Leung, Tang, \& Komura, 2011; Kovacs \& Shea, 2011; Lammfromm \& Gopher, 2011; Todorov et al., 1997; Varoqui, Froger, Pélissier, \& Bardy, 2011). For example, faster learning of (intrapersonal) interlimb and postural coordination has been demonstrated when giving to the participant real-time visual information about the coordination (Faugloire, Bardy, \& Stoffregen, 2006; Kovacs \& Shea, 2011; Swinnen, Lee, Verschueren, Serrien, \& Bogaerds, 1997; Varoqui et al., 2011; Verschueren, Swinnen, Dom, \& De Weerdt, 1997). In addition, adequate location of such visual feedback is also known to facilitate the learning by reinforcing information pickup. For instance, it has been demonstrated that visual interpersonal coordination depends on how people visually attend to each others' movement (Richardson, Marsh, Isenhower, Goodman, \& Schmidt, 2007; Schmidt, Richardson, Arsenault, \& Galantucci, 2007; Varlet, Coey, Schmidt, \& Richardson, 2012). The attention of participants, and whether they pick up the relevant visual information, are factors influencing the efficiency of motor coordination with other people. Accordingly, locating the visual feedback directly on the body of the virtual human to train participants to focus on the relevant information could strengthen the attentional and perceptual bases underlying coordination, thus potentially speeding up the learning of interpersonal coordination skills.

These fundamental questions were investigated in the current study on team rowing. This activity was chosen because although performance in rowing depends on several factors such as the fitness status of the rowers, their energy management, and their technique (Baudouin \& Hawkins, 2002, 2004; Garland, 2005; Hill, 2002; Hill \& Fahrig, 2009; Smith \& Spinks, 1995), the individual skills of elite rowers are often equivalent and the difference in performance between two teams strongly depends on the ability of the athletes to row together in a highly synchronized way during the race (Hill; Ishiko, 1971; Ishiko, Katamoto, \& Maeshima, 
1983; Schneider, Angst, \& Brandt, 1978). When rowing as a crew, rowers not only have to produce as much force as possible on the oars, but they must also adapt their movements to each other. For the same individual movement patterns, the highest speed of the boat is obtained when movements of the rowers in the team are synchronized. Unsynchronized movements cause additional displacements of the boat, increasing the friction and thus decreasing its speed, especially for higher rates of movement (Hill; Williams, 1967).

With expertise, team rowers become and learn how to be synchronized. However, the learning of team rowing coordination requires time because each rower has a preferred movement pattern (Hill, 2002; Schneider et al., 1978; Wing \& Woodburn, 1995). In addition, due to individual schedule and weather conditions, outdoor team training is more the exception than the rule, and training sessions are often individualized and performed on indoor rowing machines. Altogether, it is difficult for athletes to improve their interpersonal coordination skills. Virtual reality technologies are improving rapidly and recent research has evidenced the possibility of transferring outdoor rowing conditions onto indoor rowing machines (Filippeschi et al., 2009; Fothergill, 2010; Frisoli et al., 2008; von Zitzewitz et al., 2008). However, the implementation of team-rowing learning protocols with related-coordination feedback would build interest in those machines, because one of the main limitations in outdoor conditions, both for coach and rowers, is to have an accurate estimation of the interpersonal coordination. Efficient feedback is difficult to provide to trainees.

Off-line videos can be used to obtain an accurate estimation of the synchronization, but they cannot be given as real-time feedback. Consequently, the use of virtual reality, associated with a real-time motion-capture system, may be of particular interest to provide the trainee with accurate and real-time feedback about team rowing interpersonal coordination, directly located on the virtual body in order to facilitate information pickup.

By using the combination of virtual-reality rendering and real-time motion capture, we investigated, in the current study, whether it is possible for novice rowers to learn the specific interpersonal coordination skill with a virtual teammate on an indoor rowing machine, and to transfer the acquired skill to a new synchronizing situation with a real teammate. In the learning protocol, the synchronization was either spontaneous (no feedback other than the presence of a virtual human), or increased by a visual feedback displayed on the virtual human body, giving real-time information about their coordination. We expected that (1) participants would improve their ability to synchronize with the virtual teammate, that (2) this learning would be better for the participants who had the increased feedback available, and that (3) it would help them when synchronizing with a real teammate in a transfer task.

\section{Method}

\section{I Participants}

Sixteen adults volunteered to participate in the experiment. Their mean age was $21.4(S D=2)$. All of them were recruited from the Sport Sciences Faculty at Montpellier-1 University, and thus, had good-to-high levels of physical fitness. They were all novices in the practice of outdoor and indoor rowing and none of them had any previous experience with virtual reality. All reported normal or corrected-to-normal vision and no history of color-blindness or movement disorders. Participants were randomly divided into the control and feedback groups of eight participants ( 1 female and 7 males). All participants provided informed consent prior the experiment approved by the local Ethics Committee conforming to the Declaration of Helsinki.

\subsection{Materials}

We used two Concept 2 model D indoor rowing ergometers placed one behind the other. A Samsung 40in LCD monitor was positioned in front of the forward ergometer right above its flywheel (see Figure 1). The monitor displayed a boat in outdoor conditions with a virtual teammate rowing at the front (see Figure 1[b]). The view was egocentric, showing the participant rowing inside the boat on the seat right behind the virtual teammate. Movements of the virtual teammate corresponded to the recorded actions of a real rower who participated in national Italian rowing competitions and who did not 


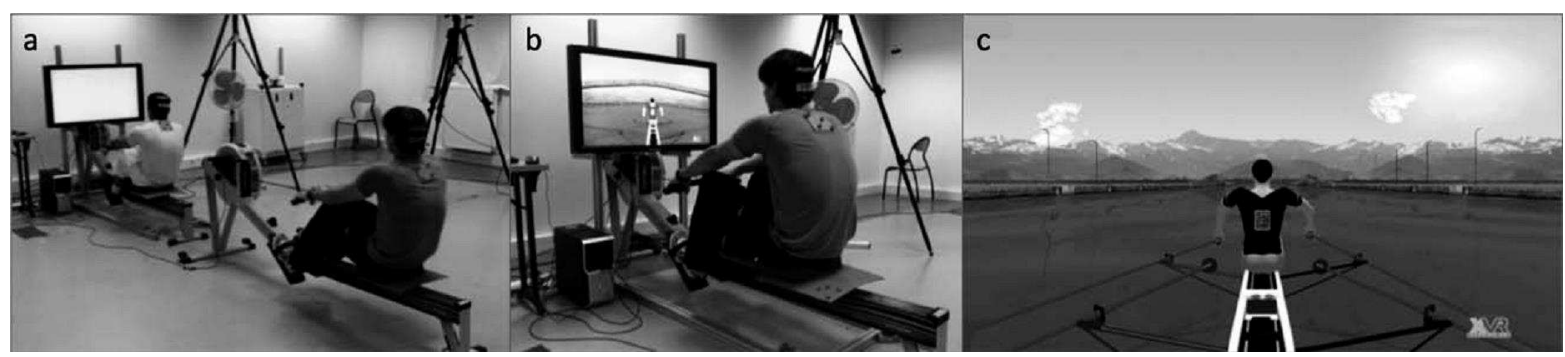

Figure I. Experimental setup. (a) Coordination with the real teammate in the transfer condition. (b) Coordination with the virtual teammate in the other conditions. (c) Virtual environment displayed on the monitor.

participate in the experiment as a confederate. They were recorded prior to the study at the rates of 18,24 , and $30 \mathrm{rpm}$ using the SPRINT platform (see Filippeschi et al., 2009). In the transfer task, the monitor only displayed a small flashing square in order to give the tempo (i.e., 18, 24, or $30 \mathrm{rpm}$ ) to the real teammate (confederate) rowing on the front ergometer (see Figure 1[a]). In the transfer task, both the participant and the confederate used headphones with white noise to prevent them from hearing each other rowing because it might have aided synchronization compared to the virtual-teammate conditions, in which no auditory information was available. The rowing simulation was controlled using the MATLAB Simulink, whereas the graphic display was managed by an application based on VRMedia's XVR software.

The front-to-back movements of the handle and the seat, and the angular motion of the participant's trunk, were selected as the most representative variables of the rowing gesture. They were collected at a sampling rate of $100 \mathrm{~Hz}$ by using eight Vicon MXl3 infrared cameras. The collected time series were recorded and used in real time in order to (1) update the positions of the oars, the seat, and the point of view of the participant in the virtual environment, and (2) compute an index of synchronization between the movements of the participant and the teammate, ranging from 0 (no synchronization) to 1 (perfect synchronization). The index of synchronization corresponded to the average of the absolute differences between the handle, seat, and trunk movements of the teammate and the participant. To correctly compute this index, the movements' amplitude of the teammate was normalized to the movements' amplitude of participants before every session by using pre-trials recorded at different frequencies. The value of the index was used for real-time updates of the color of the virtual human between red (no synchronization) and green (perfect synchronization), as necessary. The index was mapped to a normalized RGB color representation in which red is $(1,0,0)$ and green is $(0,1,0)$. These values were obtained by linear interpolation between these two values. At the last stage of the visualization, the normalized floatingpoint colors were converted to 8-bit as part of the 3D visualization of the virtual environment. This resulted in 256 levels, which provided an accurate and continuous feedback.

The end-to-end latency of the system from the motion capture by the Vicon system to the visualization on the corresponding changes on the screen was initially estimated in the range of $21-41 \mathrm{~ms}$ by internally measuring the delay between the data received from the Vicon system and the corresponding graphic frame displayed on the display after vertical synchronization. This latency included the sum of the delays of all component systems, Vicon, Simulink, and 3D rendering on the screen. In order to further reduce this latency, we modified the scheduling of the communication between the data processing component in Simulink and the graphical display in XVR, taking into account the vertical synchronization wait time. With this technical improvement, the system latency finally ranged in the interval of 11-29 ms.

\subsection{Procedure}

The duration of the learning protocols was 11 days. Participants performed the pre-, post- and reten- 


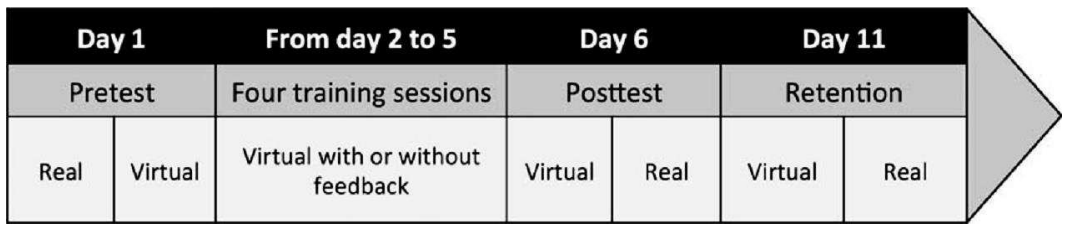

Figure 2. Experimental design for the participants of the control and feedback groups.

tion tests the first, sixth and eleventh day, respectively, and the learning sessions from the second to the fifth day (see Figure 2).

Pretests, posttests, and retention tests evaluated the ability of participants to coordinate with a real teammate and with a virtual teammate. Rowing on the ergometer, participants were instructed to do their best throughout the trial to coordinate with the movements of the real teammate (i.e., the confederate on the ergometer ahead) in the transfer condition, and with the movements of the virtual teammate displayed on the monitor in the other conditions. The same instructions were given to the participants for the learning sessions, with the exception that participants from the feedback group were informed that the color of the virtual teammate would change continuously in real time between red and green as a function of the synchronization.

The participants performed two trials at each rate (18, 24 , and $30 \mathrm{rpm}$ ) for the pretests, posttests, and retention tests, and four trials at each rate $(18,24$, and $30 \mathrm{rpm})$ during the learning sessions. The duration of the trial was $90 \mathrm{~s}$, which was comfortable for all participants and did not produce significant fatigue at the three movement frequencies. The order of the three movement rates was randomized for all participants. At the beginning of each session, the participants performed three trials without a teammate when rowing at the three selected frequencies, which were used as a warm-up as well as to normalize the movements' amplitude of the participant and the teammate in order to correctly compute the index of synchronization in real time. Two males of similar size were used as confederates and each participant performed the pretests, posttests, and retention tests with the same confederate. The two confederates were used for the same number of participants in the control and feedback groups.

\subsection{Data Analysis}

We discarded the first three strokes of each trial to eliminate transient behavior. The front-to-back movements of the handle and the seat, and the angular motion of the trunk of participants, were low-pass filtered using a $10-\mathrm{Hz}$ Butterworth filter. In line with previous research that investigated rhythmic interpersonal coordination, we computed the continuous relative phase between the front-to-back movements of the handle and the seat, and the angular motion of the trunk of the participant and the teammate in order to examine the coordination produced (Richardson et al., 2007; Schmidt, Carello, \& Turvey, 1990; Schmidt \& Richardson, 2008; Varlet, Marin, Lagarde, \& Bardy, 2011). We calculated the relative phase as

$$
\phi(t)=\theta_{1}(t)-\theta_{2}(t),
$$

where $\theta_{1}(t)$ and $\theta_{2}(t)$ were the phase angles of the teammate and the participant, respectively, computed as

$$
\theta(t)=\arctan \frac{\dot{x}(t)}{x(t)}
$$

where $\dot{x}(t)$ was the velocity (normalized in terms of the mean angular frequency of each half-cycle; see Varlet \& Richardson, 2011, for details) and $x(t)$ was the position (Kelso, 1995). Based on these values, the phase shift from the intended coordination was computed. Using the teammate as the reference, negative and positive phase shift values indicate that the movements of the participants lead $(-\theta)$ and lag $(+\theta)$ those of the team mate, respectively. We also computed the $S D$ of the relative phase to examine the variability of the coordination produced (Richardson et al., 2007; Schmidt et al., 2007). Finally, because the performance of the team also depends on the power output of the rowers, we 
extracted the power output of participants at each stroke and computed the average of the trial.

\subsection{Statistical Analysis}

We used $3 \times 2 \times 3 \times 2$ mixed-model ANOVAs with Test (pretest, posttest, and retention), Teammate (Real and Virtual), Frequency (18, 24, and $30 \mathrm{rpm}$ ), and Group (Control and Feedback) as factors for the statistical analysis of the phase shift, the $S D$ of the relative phase and average power output. Newman-Keuls post-hoc comparisons were used when necessary to determine the origin of the effect.

\section{Results}

We examined in the current study (1) whether it is possible for rowers to learn interpersonal coordination when training with a virtual teammate, $(2)$ whether practice is more efficient when training with real-time, coordination-related visual feedback, and (3) how it could help to better coordinate with a real teammate in a transfer task. We tested learning protocols in virtual reality with or without real-time information about the coordination (feedback or control group) by comparing the coordination of participants with a teammate (virtual and real) in pretests, posttests, and retention tests. Examination of the movements collected revealed that the angular motion of the trunk was sometimes aperiodic, with no clear preferred frequency, and with a very low signal-to-noise ratio. In such a case it was almost impossible to compute the relative phase between the two trunks. These aperiodic movements occurred because participants tended to sometimes row without moving their trunks, a behavior that is often observed with novice rowers and that might have been strengthened in our experiment because they were instructed to focus on an interpersonal coordination task. This was often observed (i.e., for at least five of the participants of each group) Therefore, we decided to perform the analyses on the coordination between the trainee and the teammate by taking into account the movements of the seats and the hands only.

\section{I Phase Shift}

3.I.I Coordination of the Hands. The ANOVA performed on the phase shift between the movements of the hands of the participants and the teammate yielded significant main effects for Test, $F(2,28)=7.03$, $p<.005, \eta_{p}^{2}=0.33$, Teammate, $F(1,14)=424.09$, $p<.001, \eta_{p}^{2}=0.97$, and Frequency, $F(2,28)=49.68$, $p<.001, \eta_{p}^{2}=0.78$. These effects indicated that lower phase shifts values were obtained when the teammate was real compared to virtual, with lower movement frequencies (all frequencies significantly different, $p<$ $.005)$, and in posttests and retention tests compared to the pretest, $p<.005$ and $p=0.02$, respectively (see Figures 3 and 4 ).

The ANOVA also yielded a significant interaction between Teammate and Frequency, $F(2,28)=19.91$, $p<.001, \eta_{p}^{2}=0.59$, showing that the larger lags observed for higher frequencies were stronger with the virtual teammate compared to the real teammate (i.e., all frequencies different for the virtual teammate, $p<.001$ ), whereas the difference between 18 and $24 \mathrm{rpm}$ was not significant for the real teammate, $p=.07$ (see Figure 5 ). Finally, a significant interaction between Group and Test was revealed, $F(2,28)=3.73, p=.04, \eta_{p}^{2}=0.22$, indicating lower phase shift values for the feedback group compared to the control group in posttest, $p=0.04$, showing stronger learning when training with the feedback. No other effects were significant for this analysis (i.e., all $p$ values $>$.l)

3.1.2 Coordination of the Seats. Confirming the results observed at the hand-level interpersonal coordination, the ANOVA performed on the phase shift between the movement of the seats yielded significant main effects for Teammate, $F(1,14)=222.73, p<$ $.001, \eta_{p}^{2}=0.94$, and for Frequency, $F(2,28)=57.67$, $p<.001, \eta_{p}^{2}=0.80$. In addition, a significant interac tion was found between these two factors, $F(2,28)=$ $24.17, p<.001, \eta_{p}^{2}=0.63$. In contrast to the analysis at the level of the hands, although the ANOVA yielded a significant main effect for Test, $F(2,28)=3.63$, $p=.04, \eta_{p}^{2}=0.20$, posthoc tests only revealed lower phase shift values at the posttest compared to the pretest, 

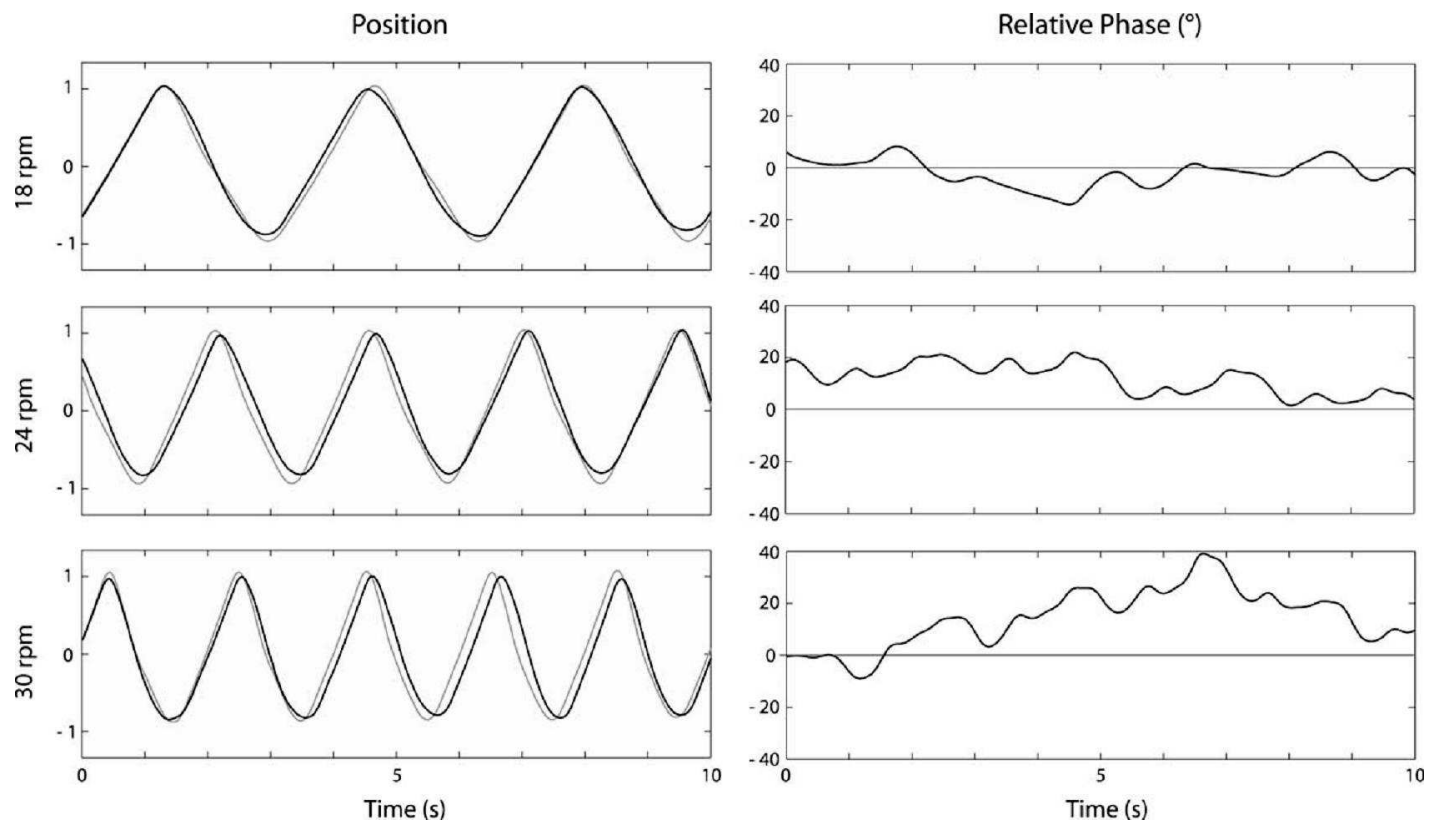

Figure 3. Movements of the hands of a representative participant (black) coordinating with a real teammate (gray) during the last $10 \mathrm{~s}$ of the 18,24, and $30 \mathrm{rpm}$ trial and the corresponding continuous relative phase, illustrating the lag increase of participants with faster movement frequency.

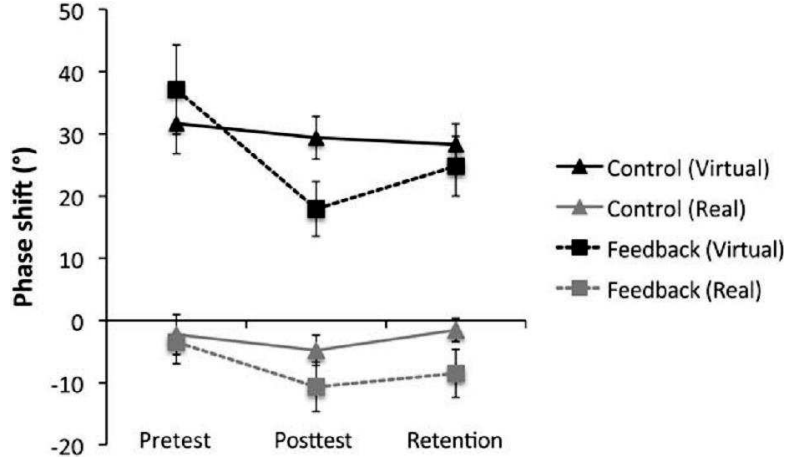

Figure 4. Phase shift values (averaged for the three movement frequencies) between movements of the hands obtained with the real (gray) and virtual (black) teammate in the pretests, posttests, and retention tests for the feedback (square and dashed) and control (triangle) groups. Error bars represent standard error.

$p=.03$. Moreover, the Group $\times$ Test interaction did not reach significance, $F(2,28)=2.19, p=.13, \eta_{p}^{2}=$ 0.14 , showing no effect of the feedback at the level of the seats. This analysis did not reveal any other significant effect (all $p$ values $>0.1$ ).

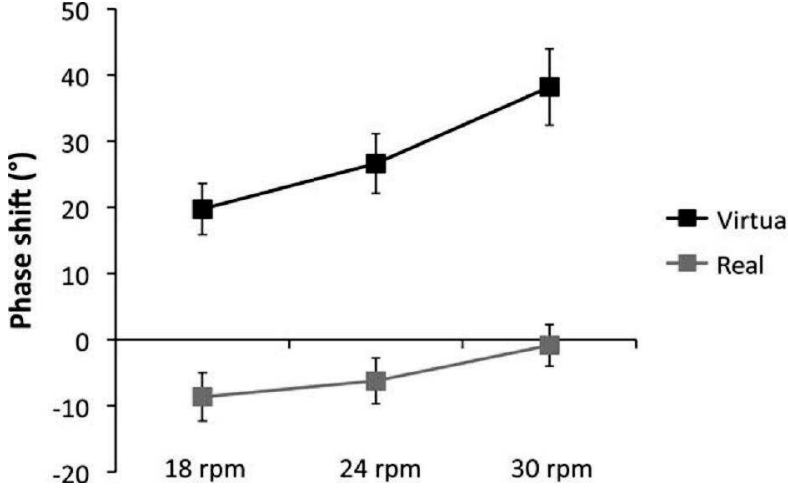

Figure 5. Phase shift values between movements of the hands obtained with the real (gray) and virtual (black) teammate as a function of movement frequency. Error bars represent standard error

Overall, these results show that the movements of participants lagged those of the teammates more importantly for higher movement frequencies and when the teammate was virtual compared to real. In addition, the learning sessions in virtual reality allowed reducing this lag for both virtual and real teammates, an effect (at the 


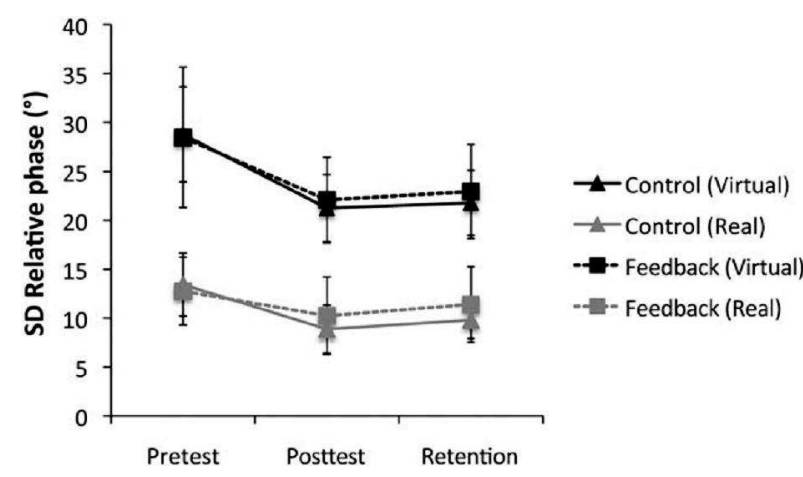

Figure 6. SD of the continuous relative phase (averaged for the three movement frequencies) between the movements of the hands obtained with the real (gray) and virtual (black) teammate in the pretest, posttests, and retention tests for the feedback (square and dashed) and control (triangle) groups. Error bars represent standard error.

level of the hands) stronger when real-time feedback was available and which was preserved at the retention test.

\subsection{Standard Deviation of the Relative Phase}

\subsection{Coordination Variability at the Hand}

Level. The ANOVA performed on the standard deviation of the relative phase at the level of the movements of the hands yielded significant main effects for Test, $F(2,28)=9.82, p<.001, \eta_{p}^{2}=0.41$, Teammate, $F(1,14)=652.66, p<.001, \eta_{p}^{2}=0.98$, Frequency, $F(2,28)=9.63, p<.001, \eta_{p}^{2}=0.41$, and a significant interaction between Test and Teammate, $F(2,28)=$ $13.57, p<.001, \eta_{p}^{2}=0.49$, indicating a lower variability when coordinating with the real teammate compared to the virtual teammate, at the rates of 24 and $30 \mathrm{rpm}$, compared to $18 \mathrm{rpm}$ (all $p$ values $<.005$ ), and in post tests and retention tests compared to the pretest for both virtual (all $p$ values $<.001$ ) and real teammates (all $p$ values $<.005$; see Figure 6 ). The post-hoc comparisons did not reveal any difference between posttests and retention tests for both real and virtual teammates (all $p$ values $>$ 4 ), showing that the performance improvement was maintained in retention tests. No other effects were significant for this analysis (all $p$ values $>.1$ ).

\subsubsection{Coordination Variability at the Seat}

Level. In line with the hands analysis, the ANOVA performed on the $S D$ of the relative phase between the movements of the seats yielded significant main effects for Test, $F(2,28)=13.10, p<.001, \eta_{p}^{2}=0.48$, Teammate, $F(1,14)=710.70, p<.001, \eta_{p}^{2}=0.98$, Frequency, $F(2,28)=14.57, p<.001, \eta_{p}^{2}=0.51$, and a significant interaction between Test and Teammate, $F(2,28)=18.52, p<.001, \eta_{p}^{2}=0.57$. Post-hoc comparisons demonstrated the same significant differences at the level of the seats and the hands. Collectively, these results show that it was more difficult for participants to maintain a stable coordination when synchronizing with the virtual teammate compared to the real teammate and at the slowest frequency, and that the learning protocol allowed participants to improve their interpersonal coordination with the virtual teammate but also with the real teammate in the transfer task.

\subsection{Power Output}

The ANOVA performed on the average power output yielded significant main effects for Test, $F(2,28)$ $=7.12, p<.005, \eta_{p}^{2}=0.34$, Teammate, $F(1,14)=$ $7.24, p=0.02, \eta_{p}^{2}=0.34$, and Frequency, $F(2,28)=$ $151.40, p<.001, \eta_{p}^{2}=0.92$, as well as significant interactions between Frequency and Teammate, $F(2,28)=$ $25.25, p<.001, \eta_{p}^{2}=0.64$, and between Frequency and Test, $F(4,56)=8.32, p<.001, \eta_{p}^{2}=0.37$. Posthoc comparisons showed that the power output obviously increased for higher rates of movement as indicated by significant differences between the three frequencies (all $p$ values $<.001$ ); and more importantly, that, for the movement rates of 24 and $30 \mathrm{rpm}$, the power output of participants was lower when synchronizing with the virtual teammate compared to the real teammate $(p=.01$ and $\mathrm{p}<.001$, respectively) and in posttests and retention tests compared to the pretest (all $p$ values $<.001)$. These results show that the difficulty to coordinate with the virtual teammate and the improvements of the coordination in posttests and retention tests at the highest frequencies were associated with a decrease of the power output of participants. 


\section{Discussion}

The goal of the current study was to investigate, using the example of team rowing, how it is possible to (1) learn interpersonal coordination when training with a virtual teammate, (2) accelerate the acquisition of this coordinative pattern with real-time visual feedback, and (3) transfer the skill of interpersonal coordination to synchronizing situations with a real teammate. Our results show that participants were able to coordinate and improve their coordination with both the virtual and real teammates, and that this improvement was better for the participants who received the feedback. These points are discussed below as well as possible improvements for indoor rowing machines using virtual reality.

Our participants were able to coordinate their movements with those of both virtual and real teammates at the different movement rates. However, lower perform ance occurred when participants coordinated with the virtual teammate compared to the real teammate, as indicated by an increase in movement lag and in the variability of coordination. An agency effect is a strong potential cause for this difference, in line with previous research showing that our movements are more influenced by those of a real human compared to those of nonhuman agents such as a virtual human or a robot (Blakemore \& Frith, 2005; Kilner et al., 2003; Press et al., 2005, Stanley et al., 2007). It is probable that the difference observed between the real and virtual teammates was due to this agency effect, and more specifically, to the involvement of different control processes, which calls for further effort to develop a more human appearance of the virtual teammate.

Complementary factors in the current experiment might have also contributed to this result. First, although reduced as much as possible, the end-to-end latency of our system might have played a role in the difference obtained between virtual and real conditions, and more specifically, in the increased movement lag observed when synchronizing with the virtual teammate. The latency of our system, 11-29 ms, might have artificially increased the phase shifts up to $3.13,4.18$, and $5.22^{\circ}$ (values computed for a latency of $29 \mathrm{~ms}$ ), for the 18,24 , and $30 \mathrm{rpm}$ movement frequencies, respectively.
Although it is not negligible, it remains minor compared to the values experimentally observed (see Figure 5), and therefore cannot explain in itself the differences observed between the real and virtual teammates. Although they were both biological, the movements of the virtual teammate had more variability and different kinematics compared to the one of confederates, because this movement was recorded on the SPRINT rowing machine similar to outdoor machines (i.e., two oars instead of a handle; see Filippeschi et al., 2009). This might have decreased the predictability of the movement, and thus led participants to adopt a reactive rather than an anticipatory behavior, as indicated by greater positive phase shift values. Finally, the size of the virtual teammate, which was smaller than the real teammate, might also have contributed to the difference in performance between the real and virtual conditions, as coordination with a smaller partner may be slightly more demanding. Greater attention might be necessary to detect on a smaller teammate the relevant movement information ensuring stable coordination.

Taken together, our results show that it is possible for trainees to improve their ability to coordinate with other people, even in virtual reality. Using an appropriate learning protocol, participants improved their interpersonal coordination as indicated by a decrease in how their movements lagged those of the teammate and in the variability of the coordination. Interestingly, these improvements also occurred in the transfer task, showing that although participants learned to coordinate with a virtual teammate, they became better when requested to coordinate with a real teammate, an improvement preserved at the retention test, which demonstrates the robustness of the learning effect. Nevertheless, the effectiveness of the transfer might need further comparisons, for instance, with an additional control group performing no learning at all. Here, the assumption is that our participants were better synchronized with the real team mate in the posttest only because they performed the task twice. However, our participants actually performed the task three times with a five-day interval between them (pretest, posttest, and retention test), and the performance at the retention test did not become better, which goes against this assumption. Collectively, these results show, we believe for the first time, how virtual 
reality can help learning interpersonal coordination, which more generally supports previous findings showing the interest of virtual reality for the acquisition of new perceptuomotor skills (Lammfromm \& Gopher, 2011; Ruffaldi et al., 2011; Swinnen et al., 1997; Todorov et al., 1997).

Our results also demonstrate that it is possible to accelerate the learning by associating virtual reality with standard motion-capture systems in order to give realtime information about their interpersonal coordination. The participants who received the feedback during the learning session coordinated with the real and virtual teammates in posttests and retention tests with smaller phase lags. Giving real-time information about the coordination speeded up the learning process, a result in line with previous studies that investigated the learning of other kinds of coordination such as interlimb or postural coordination (Faugloire et al., 2006; Swinnen et al., 1997; Varoqui et al., 2011; Verschueren et al., 1997). However, it is possible that better improvements of the feedback group were not only due to the availability of information about the coordination but also to an increase in the perceptual capacity of our participants. As initially assumed, displaying the visual feedback directly on the body of the virtual teammate might have helped participants to improve their capacity to visually focus on the movements of the teammate, and thus, to have better coordination (Richardson et al., 2007; Schmidt et al., 2007; Varlet, Coey, et al., 2012). This result calls for testing this assumption in future research by using other perceptual modalities to give real-time information about the coordination with auditory or tactile feedback (e.g., Kelso, Fink, DeLaplain, \& Carson, 2001; Lagarde \& Kelso, 2006; Ruffaldi et al., 2009; Varlet, Marin, Issartel, Schmidt, \& Bardy, 2012; von Zitzewitz et al., 2008; Zelic, Mottet, \& Lagarde, 2012). Our results give motivation to compare the performance of the feedback group with the performance of a control group only learning with a real teammate, in order to determine whether virtual reality practice with adequate feedback can match or surpass real practice alone.

More specifically, for the rowing activity, our results show that even during indoor individual practice it is possible for rowers to improve the ability to coordinate with a teammate by using a simple virtual-reality device. In view of the difficulty and the duration needed to learn team rowing coordination in classical outdoor training (Hill, 2002; Schneider et al., 1978), such an indoor system with adequate feedback may be very useful for rowers, irrespective of outdoor conditions. Moreover, although the generalizability of these results for elite rowers will need further research, it can be assumed that they might be more likely to take advantage of such a system because they are already familiar with the rowing skill and do not have to focus on their individual (intrapersonal) coordination. However, in view of the current results, the feedback used in this experiment will need to be further adapted to the specificities of the rowing activity in order to further accelerate the learning. Our results show that the participants of the feedback group coordinated with their teammate in posttests and retention tests with smaller phase lags. This result demonstrates an improvement of the control processes of the trainees, allowing a stronger anticipation of the movements of the teammate. Although such anticipation is of particular interest in a variety of interpersonal activities, it can become detrimental to rowing performance because it can decrease the synchrony between rowers. Accordingly, the feedback will need to be adapted to avoid such an issue.

Moreover, although participants improved their interpersonal coordination, our results also show a decrease of their power output in posttests and retention tests. To be coordinated with the teammate, the participants changed their intrinsic rowing technique, affecting the power output, which is not optimal for performance (Baudouin \& Hawkins, 2002; Hill, 2002). These changes of the intrinsic technique of participants, and thus, of their intrapersonal coordination (i.e., coordination of their different body segments), when synchronizing with the teammate are in accordance with the close relationship between intrapersonal and interpersonal coordination demonstrated in other studies (Coey, Varlet, Schmidt, \& Richardson, 2011; Ramenzoni, Davis, Riley, Shockley, \& Baker, 2011; Varlet, Marin, et al., 2011). However, it is possible that such an effect could have been reduced in the current study with longer learning sessions. In line with previous research, partici- 
pants may have changed their intrapersonal coordination to coordinate with the teammate by reducing the degrees of freedom to control, and could have recovered a more efficient intrapersonal coordination with longer learning protocols (Temprado, Della-Grasta, Farrell, \& Laurent, 1997; Vereijken, Van Emmerik, Whiting, \& Newell, 1992; Vereijken, Van Emmerik, Bongaardt, Beek, \& Newell, 1997). In addition, to provide further information about the coordination, future feedback may also be designed to constrain the power output of participants to avoid such an effect.

Finally, for the rowing activity, the learning protocol and the given feedback in this study allowed participants to better (visually) coordinate their movements with those of a teammate. However, in outdoor conditions, the perceptual support underlying the coordination between rowers is intrinsically multimodal. Rowers regulate their movements to maintain the coordination by using visual information but also other perceptual information originating from the auditory, haptic, or tactile systems. Although increasing the capacity of rowers to use visual information is expected to result in enhanced outdoor rowing performance, it encourages the development of future indoor rowing machines that improve the use of all modalities, and potentially the relation between them (e.g., Stoffregen \& Bardy, 2001), in order to optimize learning.

To conclude, the current study demonstrates with the example of team rowing that it is possible to learn interpersonal coordination when training with a virtual teammate, to transfer the learned skill to coordination with a real human, and to speed up the learning with real-time coordination-related feedback. These results demonstrate the interest of virtual reality as a tool to learn to coordinate with other people. They thus open new perspectives for improving performance in rowing but also in a variety of interpersonal activities.

\section{Acknowledgments}

This research was supported by SKILLS, an Integrated Project (FP6-IST contract \#035005) of the Commission of the Europcan Community.

\section{References}

Baudouin, A., \& Hawkins, D. (2002). A biomechanical review of factors affecting rowing performance. British Journal of Sports Medicine, 36(6), 396-402.

Baudouin, A., \& Hawkins, D. (2004). Investigation of biomechanical factors affecting rowing performance. Journal of Biomechanics, 37(7), 969-976.

Bernieri, F. J. (1988). Coordinated movement and rapport in teacher-student interactions. Journal of Nonverbal Behavior, 12(2), 120-138.

Bernieri, F. J., Davis, J. M., Rosenthal, R., \& Knee, C. R. (1994). Interactional synchrony and rapport: Measuring synchrony in displays devoid of sound and facial affect. Personality and Social Psychology Bulletin, 20(3), 303-311.

Bidcau, B., Kulpa, R., Mćnardais, S., Fradet, L., Multon, F., Delamarche, P., \& Arnaldi, B. (2003). Real handball goalkeeper vs. virtual handball thrower. Presence: Teleoperators and Virtual Environments, 12(4), 411-421.

Bideau, B., Kulpa, R., Vignais, N., Brault, S., Multon, F., \& Craig, C. (2010). Using virtual reality to analyze sports performance. IEEE Computer Graphics and Applications, 14-21.

Blakemore, S. J., \& Frith, C. (2005). The role of motor contagion in the prediction of action. Neuropsychologia, 43(2), 260-267.

Brune, M., Sonntag, C., Abdel-Hamid, M., Lehmkamper, C., Juckel, G., \& Troisi, A. (2008). Nonverbal behavior during standardized interviews in patients with schizophrenia spec trum disorders. Journal of Nervous er Mental Disease, 196(4), 282-288.

Chan, J. C., Leung, H., Tang, J. K., \& Komura, T. (2011). A virtual reality dance training system using motion capture technology. IEEE Transactions on Learning Technologies, 4(2), 187-195.

Chartrand, T. L., \& Bargh, J. A. (1999). The chameleon effect: The perception-behavior link and social interaction. Journal of Personality and Social Psychology, 76(6), 893-910.

Coey, C., Varlet, M., Schmidt, R. C., \& Richardson, M. J. (2011). Effects of movement stability and congruency on the emergence of spontaneous interpersonal coordination. Experimental Brain Research, 211, 483-493.

Faugloire, E., Bardy, B. G., \& Stoffregen, T. A. (2006). Dynamics of learning new postural patterns: Influence on preexisting spontaneous behaviors. Journal of Motor Behavior, 38(4), 299-312.

Filippeschi, A., Ruffaldi, E., Frisoli, A., Avizzano, C. A., Varlet, M., Marin, L., Lagarde, J., Bardy, B. G., Bergamasco, M. 
(2009). Dynamic models of team rowing for a virtual environment rowing training system. The International Journal of Virtual Reality, 4(8), 19-26.

Fothergill, S. (2010). Examining the effect of real-time visual feedback on the quality of rowing technique. Procedia Engineering, 2(2), 3083-3088.

Frisoli, A., Ruffaldi, E., Bagnoli, L., Filippeschi, A., Avizzano, C. A., Vanni, F., \& Bergamasco, M. (2008). Preliminary design of rowing simulator for in-door skill training. Proceedings of the 2008 Ambi-Sys Workshop on Haptic User Interfaces in Ambient Media Systems, Article 9.

Garland, S. W. (2005). An analysis of the pacing strategy adopted by elite competitors in $2000 \mathrm{~m}$ rowing. British Journal of Sports Medicine, 39(1), 39-42.

Hill, H. (2002). Dynamics of coordination within elite rowing crews: Evidence from force pattern analysis. Journal of Sports Sciences, 20(2), 101-117.

Hill, H., \& Fahrig, S. (2009). The impact of fluctuations in boat velocity during the rowing cycle on race timc. Scandinavian Journal of Medicine $\mathfrak{F}$ Science in Sports, 19(4), 585594.

Hove, M. J., \& Risen, J. L. (2009). It's all in the timing: Interpersonal synchrony increases affiliation. Social Cognition, $27(6), 949-960$

Ishiko, T. (1971). Biomechanics of rowing. Biomechanics $I I$, 249-252

Ishiko, T., Katamoto, S., \& Maeshima, T. (1983). Analysis of rowing movements with radiotelemetry. Proceedings of the Eighth International Congress of Biomechanics (pp. 816-21). Champaign, IL: Human Kinetics.

Kclso, J. A. S. (1995). Dynamic patterns: The self-organization of brain and behavior. Cambridge, MA: MIT Press.

Kelso, J. A., Fink, P. W., DeLaplain, C. R., \& Carson, R. G. (2001). Haptic information stabilizes and destabilizes coordination dynamics. Proceedings of the Royal Society of London. Series B: Biological Sciences, 268(1472), 12071213.

Kilner, J. M., Paulignan, Y., \& Blakemore, S. J. (2003). An interference effect of obscrved biological movement on action. Current Biology, 13(6), 522-525.

Kovacs, A. J., \& Shea, C. H. (2011). The learning of $90^{\circ}$ continuous relative phase with and without Lissajous feedback: External and internally generated bimanual coordination. Acta Psychologica, 136, 311-320.

Kupper, Z., Ramseyer, F., Hoffmann, H., Kalbermatten, S., \& Tschacher, W. (2010). Video-based quantification of body movement during social interaction indicates the sever- ity of negative symptoms in patients with schizophrenia. Schizophrenia Research, 121, 90-100.

Lagarde, J., \& Kelso, J. A. S. (2006). Binding of movement, sound and touch: Multimodal coordination dynamics. Experimental Brain Research, 173(4), 673-688.

Lakin, J. L., \& Chartrand, T. L. (2003). Using nonconscious behavioral mimicry to create affiliation and rapport. Psychological Science, 14(4), 334.

Lammfromm, R., \& Gopher, D. (2011). Transfer of skill from a virtual reality trainer to real juggling. Proceedings of the International Conference SKILLS (Vol. 1), Articlc 54.

Marsh, K. L., Richardson, M. J., \& Schmidt, R. (2009). Social connection through joint action and interpersonal coordination. Topics in Cognitive Science, I(2), 320-339.

Marsh, K. L., Richardson, M. J., Baron, R. M., \& Schmidt, R. (2006). Contrasting approaches to perceiving and acting with others. Ecological Psychology, 18(1), 1-38.

Oztop, E., Franklin, D., Chaminade, T., \& Gordon, C. (2005). Human-humanoid interaction: Is a humanoid robot perceived as a human? International Journal of Humanoid Robotics, 2, 537-559.

Press, C., Bird, G., Flach, R., \& Heyes, C. (2005). Robotic movement elicits automatic imitation. Cognitive Brain Research, 25(3), 632-640

Ramenzoni, V. C., Davis, T. J., Riley, M. A., Shockley, K., \& Baker, A. A. (2011). Joint action in a cooperative precision task: Nested processes of intrapersonal and interpersonal coordination. Experimental Brain Research, 211, 447-457

Richardson, D. C., Dale, R., \& Kirkham, N. Z. (2007). The art of conversation is coordination. Psychological Science, $18(5), 407-413$.

Richardson, M. J., Marsh, K. L., \& Baron, R. M. (2007). Judg ing and actualizing intrapersonal and interpersonal affordances. Journal of Experimental Psychology: Human Perception and Performance, 33(4), 845-859.

Richardson, M. J., Marsh, K. L., Isenhower, R. W., Goodman, J. R. L., \& Schmidt, R. C. (2007). Rocking together: Dynamics of intentional and unintentional interpersonal coordination. Human Movement Science, 26(6), 867-891.

Ruffaldi, E., Filippeschi, A., Avizzano, C. A., Bardy, B., Gopher, D., \& Bergamasco, M. (2011). Feedback, affordances, and accelerators for training sports in virtual environments. Presence: Teleoperators and Virtual Environments, 20(1), 33-46.

Ruffaldi, E., Filippeschi, A., Frisoli, A., Sandoval, O., Avizzano, C. A., \& Bergamasco, M. (2009). Vibrotactile perception assessment for a rowing training system. Third Joint Euro- 
Haptics Conference, 2009 and Symposium on Haptic Interfaces for Virtual Environment and Teleoperator Systems, World Haptics 2009, 350-355.

Schmidt, R. C., Carello, C., \& Turvey, M. T. (1990). Phase transitions and critical fluctuations in the visual coordination of rhythmic movements between people. Journal of Experimental Psychology: Human Perception and Performance, 16(2), 227-247.

Schmidt, R. C., \& Richardson, M. J. (2008). Dynamics of interpersonal coordination. In A. Fuchs \& V. Jirsa (Eds.), Coordination: Neural, behavioral and social dynamics (pp. 281-308). Heidelberg: Springer-Verlag.

Schmidt, R. C., Richardson, M. J., Arsenault, C., \& Galantucci, B. (2007). Visual tracking and entrainment to an environmental rhythm. Journal of Experimental Psychology: Human Perception and Performance, 33(4), 860-870

Schneider, E., Angst, F., \& Brandt, J. D. (1978). Biomechanics in rowing. In E. Asmussen \& K. Jorgensen (Eds.), Biomechanics VI-B (pp. 115-119). Baltimore, MD: University Park Press.

Smith, R. M., \& Spinks, W. L. (1995). Discriminant analysis of biomechanical differences between novice, good and elite rowers. Journal of Sports Sciences, 13(5), 377-385.

Stanley, J., Gowen, E., \& Miall, R. C. (2007). Effects of agency on movement interference during observation of a moving dot stimulus. Journal of Experimental Psychology: Human Perception and Performance, 33(4), 915-926.

Stoffregen, T. A., \& Bardy, B. G. (2001). On specification and the senses. Behavioral and Brain Sciences, 24(2), 195-261.

Swinnen, S. P., Lee, T. D., Verschueren, S., Serrien, D. J., \& Bogaerds, H. (1997). Interlimb coordination: Learning and transfer under different feedback conditions. Human Movement Science, 16(6), 749-785.

Temprado, J., Della-Grasta, M., Farrell, M., \& Laurent, M. (1997). A novice-expert comparison of (intra-limb) coordination subserving the volleyball serve. Human Movement Science, 16(5), 653-676.

Tickle-Degnen, L., \& Rosenthal, R. (1990). The nature of rapport and its nonverbal correlates. Psychological Inquiry, I(4), 285-293.

Todorov, E., Shadmehr, R., \& Bizzi, E. (1997). Augmented feedback presented in a virtual environment accelerates learning of a difficult motor task. Journal of Motor Behavior, 29(2), 147-158.

Varlet, M., Coey, C. A., Schmidt, R. C., \& Richardson, M. J. (2012). Influence of stimulus amplitude on unintended visuomotor entrainment. Human Movement Science, 31(3), 541-552.

Varlet, M., Marin, L., Lagarde, J., \& Bardy, B. G. (2011). Social postural coordination. Journal of Experimental Psychology: Human Perception and Performance, 37(2), 473483.

Varlet, M., Marin, L., Issartel, J., Schmidt, R. C., \& Bardy, B G. (2012). Continuity of visual and auditory rhythms influences sensorimotor coordination. PLoS ONE, 7(9): e44082. doi:10.1371/journal.pone. 004408 .

Varlet, M., Marin, L., Raffard, S., Schmidt, R. C., Capdevielle, D., Boulenger, J. P., Del-Monte, J., \& Bardy, B. G. (2012). Impairments of social motor coordination in schizophrenia. PLoS ONE, 7(1): e29772. doi:10.1371 /journal.pone.0029772.

Varlet, M., \& Richardson, M. J. (2011). Computation of continuous relative phase and modulation of frequency of human movement. Journal of Biomechanics, 44(6), 12001204.

Varoqui, D., Froger, J., Pćlissicr, J. Y., \& Bardy, B. G. (2011). Effect of coordination biofeedback on (re)learning preferred postural patterns in post-stroke patients. Motor Control, 15, 187-205.

Vereijken, B., Van Emmerik, R. E. A., Bongaardt, R., Beek, W. J., \& Newell, K. M. (1997). Changing coordinative structures in complex skill acquisition. Human Movement Science, $16(6), 823-844$.

Vereijken, B., Van Emmerik, R. E., Whiting, H. T., \& Newell, K. M. (1992). Free(z)ing degrees of freedom in skill acquisition. Journal of Motor Behavior, 39(1), 29-39.

Verschueren, S. M., Swinnen, S. P., Dom, R., \& De Weerdt, W. (1997). Interlimb coordination in patients with Parkinson's disease: Motor learning deficits and the importance of augmented information feedback. Experimental Brain Research, 113(3), 497-508.

Vignais, N., Bideau, B., Craig, C., Brault, S., Multon, F., Delamarche, P., \& Kulpa, R. (2009). Does the level of graphical detail of a virtual handball thrower influence a goalkeeper's motor response? Journal of Sports Science and Medicine, 8, 501-508.

Vignais, N., Bideau, B., Craig, C., Brault, S., Multon, F., Delamarche, P., \& Kulpa, R. (2010). Judging the "passability" of dynamic gaps in a virtual rugby environment. Journal of Sports Science and Medicine, 1, 3.

Vignais, N., Kulpa, R., Craig, C., Brault, S., Multon, F., \& Bideau, B. (2010). Influence of the graphical levels of detail 
of a virtual thrower on the perception of the movement. Presence: Teleoperators and Virtual Environments, 19(3), 243-252.

von Zitzewitz, J., Wolf, P., Novaković, V., Wellner, M., Rauter, G., Brunschweiler, A., \& Riener, R. (2008). Realtime rowing simulator with multimodal feedback. Sports Technology, 1(6), 257-266.

Williams, J. G. P. (1967). Some biomechanical aspects of rowing. In J. G. P. Williams and A. C. Scott (Eds.), Rowing: $A$ scientific approach (pp. 81-109). London: Kaye and Ward Ltd.
Wing, A. M., \& Woodburn, C. (1995). The coordination and consistency of rowers in a racing eight. Journal of Sports Sciences, 13, 187-187.

Zaal, F. T. J. M., \& Bootsma, R. J. (2011). Virtual reality as a tool for the study of perception-action: The case of running to catch fly balls. Presence: Teleoperators and Virtual Environments, 20(1), 93-103.

Zelic, G., Mottet, D., \& Lagarde, J. (2012). Behavioral impact of unisensory and multisensory audio-tactile events: Pros and cons for interlimb coordination in juggling. PLoS ONE, 7(2): e32308. doi:10.1371/journal.pone.0032308. 